\title{
Programas de intervención para la mejora de la imagen corporal en niños de Educación Primaria
}

\author{
Intervention programs for the body image \\ improvement in Elementary School children
}

\author{
María Pilar León González*, Irene González-Martí y Onofre Ricardo Contreras Jordán
}

Departamento de Didáctica de la Expresión Musical, Plástica y Corporal. Area de Educación Física. Universidad de Castilla-La Mancha, España

\begin{abstract}
Resumen: Los problemas derivados de una imagen corporal negativa conducen al desarrollo de programas educativos que, a través de distintos contenidos, tratan de mejorar la imagen corporal de los nińos en edad escolar. El propósito de este estudio fue analizar las características y efectos de los programas más recientes para niños de Educación Primaria, a fin de conocer aquellos más eficaces. Se analizaron 21 programas, cuyos contenidos más comunes son autoestima, alfabetización mediática, alimentación saludable y actividad física. La mayoría de estos programas están basados en una metodología interactiva y un enfoque etiológico. En general, varios programas resultan efectivos en la mejora de algunos factores de riesgo, aunque se ha observado poco efecto en los insultos sobre apariencia, la insatisfacción corporal y las conductas o síntomas de desórdenes alimentarios, tanto en el post-test como en el seguimiento. Algunas limitaciones son la falta de un grupo control y una evaluación de seguimiento.
\end{abstract}

Palabras clave: imagen corporal, programa de intervención, niños.
Abstract: Problems arising from a negative body image lead to the development of educational programs that, through different contents, try to improve the school-aged-children's body image. The aim of this study was to analyze the characteristics and effects of the most recent programs for children of Elementary School, in order to know the most effective ones. Twenty-one programs were analysed, whose most common contents are self-esteem, media literacy, healthy dieting and physical activity. Most of these programmes are based on an interactive methodology and an etiological approach. In general, some programmes are effective on the improvement of some risk factors, although it has been seen little effect on body dissatisfaction and behaviors or symptoms of eating disorder, both in post-test and follow-up. Some limitations are the lack of a control group and a follow-up assessment.

Key words: body image, intervention programmes, children.
La imagen corporal (IC) es un constructo que incluye la percepción de nuestro cuerpo; la experiencia subjetiva de actitudes, pensamientos, sentimientos y valoraciones que hacemos; y el modo en que nos comportamos según nuestras cogniciones (Raich, 2001). Esta imagen que nos formamos mentalmente puede tener connotaciones positivas o negativas y repercute en el bienestar social, emocional y físico (Huttchinson \& Calland, 2011), lo cual es un motivo de preocupación social.

La adolescencia temprana es considerada el período más crítico para la IC, especialmente en niñas (Littleton \& Oilendick, 2003), aunque las repercusiones de este constructo ya se observan en edades anteriores. Según Grogan (2016), los niños de 8 años suelen mostrar niveles similares de preocupación corporal que los adultos. De hecho, generalmente la mitad de niños de Educación Primaria están insatisfechos con su cuerpo o alguna parte de éste (Smolak, 2012); conduciendo esta situación al desarrollo de programas de prevención en el ámbito educativo, cuya finalidad es mejorar la salud y reducir o impedir el desarrollo de una IC negativa y

Dirección para correspondencia [Correspodence address]: María Pilar León González. E-mail: MariaPilar.Leon@uclm.es los problemas asociados a ésta.

Un ejemplo de ellos sería el Trastorno Dismórfico Corporal (TDC), entendido como la "preocupación por uno o más defectos o imperfecciones percibidas en el aspecto físico y que no son observables o parecen sin importancia a otras personas" (APA, 2014, p. 148). A su vez, los TDC, como la Dismorfia Muscular, tienen comorbilidad con los Trastornos de la Conducta Alimentaria -TCA- (bulimia nerviosa y/o anorexia nerviosa) y alteraciones del estado de ánimo (Ferreiro, Seoane, \& Senra, 2014; González-Martí, Fernández, Hernández-Martínez, \& Contreras, 2014).

Para prevenir estos problemas, los programas deben implementarse con niños de edades tempranas, ya que como señala Raich (2001), la IC tiene sus raíces en la infancia, y es en esta época cuando los niños forman sus actitudes sobre el tamaño corporal y la IC (Damiano et al., 2015). Por consiguiente, es necesario incidir en dicha etapa mediante programas de prevención de desórdenes alimentarios, antes de que los factores de riesgo sean más profundos (Rohde et al., 2014). En este sentido, Holt y Ricciardelli (2008) consideran que los programas con niños son más efectivos que con adolescentes y adultos, ya que en edades superiores, las actitudes y conductas son más difíciles de modificar. 
La efectividad de los programas para cambiar conductas o conocimientos también podría depender de los enfoques desde los que se plantean. Algunos autores consideran que los programas deben diseñarse desde un enfoque etiológico o socio-cognitivo (Diedrichs et al., 2015; McCabe, Connaughton, Tatangelo, \& Mellor, 2017; Richardson \& Paxton, 2010), es decir, haciendo hincapié en la reducción de factores de riesgo vinculados a la IC.

En relación a los factores personales, los programas suelen incidir, entre otras cosas, en la autoestima, debido a su repercusión en el bienestar. Es sabido que una IC pobre se asocia con bajos niveles de autoestima, ya que uno mismo no se valora como persona (Raich, 2001). De hecho, al menos una tercera parte de la autoestima está marcada por la imagen que cada uno tiene de su cuerpo (Raich, 2004) y puede moderar el grado en que los sujetos internalicen los ideales de belleza sociales (Tiggemann, 2012), lo cual también supone un factor de riesgo para el desarrollo de una IC negativa (Littleton \& Oilendick, 2003). Por ello, la autoestima tiene especial trascendencia en los programas de IC, a través de los cuales debe potenciarse que los niños desarrollen una autoestima saludable basada en sus aptitudes y logros y no en función de cómo sea su apariencia (Hutchinson \& Calland, 2011).

Además de la autoestima, existen otras variables personales que influyen en la IC, como son el perfeccionismo, autoconcepto, cambios físicos (Cash, 2012) o Índice de Masa Corporal-IMC- (Smolak, 2012).

Por otro lado, destacan algunos factores socioculturales que repercuten sustancialmente en la IC. Uno de los más influyentes son los medios de comunicación, especialmente en niños de temprana edad, ya que éstos no son capaces de ignorar las continuas presiones que ejercen los medios sobre los ideales de apariencia (Hutchinson \& Calland, 2011). Por tanto, en los programas de intervención debe incidirse en la influencia y manipulación de los medios en torno a la IC, a fin de que los nińos sean conscientes y críticos con las imágenes alejadas de la realidad que se propagan.

Además de los medios, existen otros agentes que propagan los cánones de belleza e inciden en la IC. Al tratarse de niños, los familiares son quienes mayor impacto tienen en este constructo (Hutchinson \& Calland, 2011), aunque los iguales también contribuyen en gran medida (Littlenton \& Oilendick, 2003). Por ello, algunos programas incluyen como contenido las relaciones con iguales y cuentan con los padres para su puesta en práctica (Haines et al., 2006), es decir, aplican el programa desde un enfoque ecológico, teniendo en consideración el ambiente para promover cambios (Evans, Roy, Geiger, Werner, \& Burnett, 2008).

En los últimos ańos se han desarrollado numerosos programas con el fin de mejorar la IC de nińos y adolescentes, así como prevenir problemas futuros. El propósito de este estudio es analizar las características y el grado de incidencia de estos programas sobre la IC de los niños de Primaria o la etapa equivalente en otros países, es decir, niños de entre 6 y 12 años.

\section{Método}

\section{Procedimiento de búsqueda}

Se realizó una búsqueda con las siguientes palabras clave, tanto en inglés como español: IC, programa, niños, intervención e insatisfacción corporal.

\section{Criterios y proceso de selección}

Para la selección de programas se tuvieron en cuenta los siguientes criterios:

\section{Criterios de inclusión}

- Programas escolares o extraescolares para niños de entre 6 y 12 ańos.

- Artículos publicados entre 2008 y 2017, ya que los programas que datan con anterioridad fueron revisados por Holt y Ricciardelli (2008). No obstante, se incluyen tres de 2006 y uno de 2007 que no fueron analizados por ellos.

\section{Criterios de exclusión}

- Idiomas diferentes al español o inglés.

- Artículos que no estuviesen incluidos en revistas de revisión por pares.

- Artículos de programas que hayan sido evaluados con anterioridad.

- Programas únicamente de obesidad, actividad física (AF) y/o nutrición, ya que para abordar la IC, conviene contemplar más factores que influyen en su desarrollo y no centrarse únicamente en uno de ellos.

\section{Resultados}

\section{Búsqueda de programas}

Se encontraron 96 artículos de programas, de los cuales sólo 21 cumplieron con los criterios de inclusión.

Para la clasificación de los contenidos tratados en los programas, se ha atendido a las categorías usadas por Holt y Ricciardelli (2008) y establecidas por Levine y Smolak (2006). Éstas son:

1. Psicoeducativa: aumentar el conocimiento sobre IC.

2. Habilidades de resistencia sociocultural: pensamiento crítico sobre la información mediática. 
3. Habilidades saludables de control de peso: aprender prácticas saludables.

4. Habilidades para afrontar el estrés.

5. Mejora de la autoestima.

6. Mejora de la satisfacción corporal: promover la aceptación del cuerpo.
Además, se ha añadido una séptima categoría: habilidades comunicativas e interpersonales.

Las características de los programas y los resultados obtenidos tras la intervención y un tiempo después de ésta, quedan recogidos en la Tabla 1 y Tabla 2 respectivamente.

Tabla 1. Características y resultados de los programas de IC.

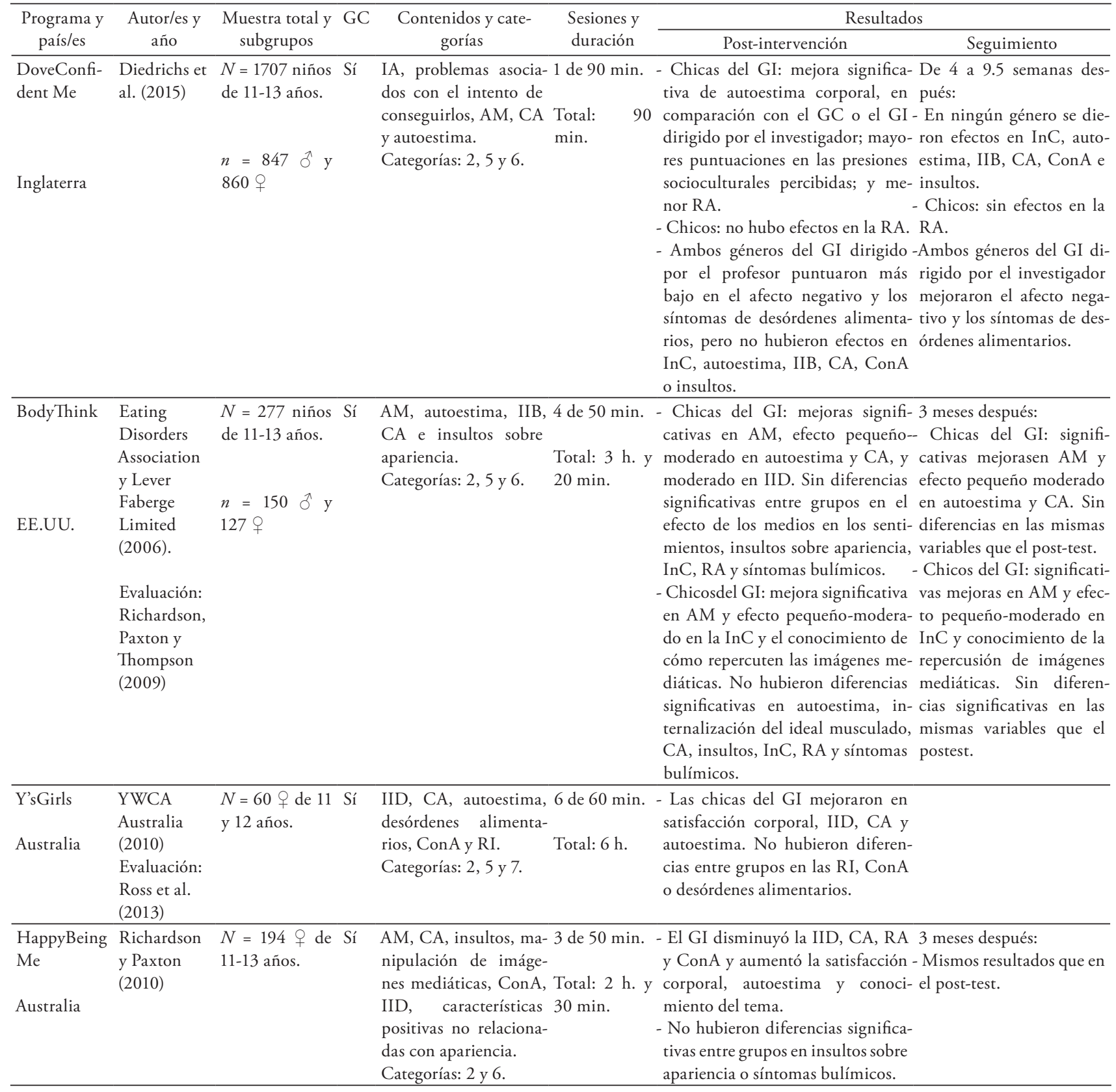




\begin{tabular}{|c|c|c|c|c|c|}
\hline \multirow{2}{*}{$\begin{array}{c}\text { Programa y } \\
\text { país/es }\end{array}$} & \multirow{2}{*}{$\begin{array}{c}\text { Autor/es y } \\
\text { año }\end{array}$} & \multirow{2}{*}{ Muestra total y GC } & \multirow{2}{*}{$\begin{array}{c}\text { Contenidos y cate- } \\
\text { gorías }\end{array}$} & \multirow{2}{*}{$\begin{array}{l}\text { Sesiones y } \\
\text { duración }\end{array}$} & Resultados \\
\hline & & & & & Post-intervención \\
\hline Healthy me & $\begin{array}{l}\text { McCabe et } \\
\text { al. (2017) }\end{array}$ & $\begin{array}{l}N=652 \text { niños Sí } \\
\text { de } 8-10 \text { años. } \\
n=321 ð \text { y } \\
331 \text { ? }\end{array}$ & $\begin{array}{l}\text { Aceptación de la diver- } \\
\text { sidad, identificación y } \\
\text { evaluación crítica de } \\
\text { las influencias en la } \\
\text { estima corporal, iden- } \\
\text { tificación de fortalezas } \\
\text { no relacionadas con la } \\
\text { apariencia, y conduc- } \\
\text { tas de AS y AF. } \\
\text { Categorías: } 2,3,5,6 \\
\text { y } 7 \text {. }\end{array}$ & $\begin{array}{l}4 \text { de } 60 \text { min y } \\
1 \text { de recapitu } \\
\text { lación } 3 \text { me } \\
\text { ses después } \\
\text { de acabar el } \\
\text { programa. } \\
\text { Total: } 5 \text { h. }\end{array}$ & $\begin{array}{l}\text { y - Ningún efecto en autoestima; es- } 3 \text { meses después: } \\
\text { 1- trategias de cambio corporal; pre-- Chicos: efecto en niveles } \\
\text { e- sión percibida de iguales, padres de AF y menor inversión } \\
\text { s o medios; índices de AF y afectos en normas masculinas. } \\
1 \text { positivos o negativos. } \\
\text { - El GI mejoró su estima muscular de fruta; la presión perci- } \\
\text { y la ingesta de fruta. } \\
\text { - Los chicos del GI redujeron su in- medios, ni en los afectos } \\
\text { versión en las normas masculinas. positivos o negativos. } \\
\text { - El GI mejoró significati- } \\
\text { vamente la autoestima y } \\
\text { estima muscular. }\end{array}$ \\
\hline Sin nombre & $\begin{array}{l}\text { Yeh, Liou } \\
\text { y Chien } \\
(2011)\end{array}$ & $\begin{array}{l}N=314 \text { nińos Sí } \\
\text { de } 10 \text { y } 11 \text { ańos. } \\
n=142 \text { ठ y } \\
172 q\end{array}$ & $\begin{array}{l}\text { IC, autoestima, AM, } \\
\text { gestión del estrés, cli- } \\
\text { ma amistoso y comu- } \\
\text { nicación interpersonal. } \\
\text { Categorías: } 2,4,5,6 \\
\text { y } 7\end{array}$ & $\begin{array}{l}8 \text { de } 40 \text { min. } \\
\text { Total: } 5 \text { h. y } \\
20 \text { min. }\end{array}$ & $\begin{array}{l}\text { - Mejoras en la satisfacción corpo- } 3 \text { meses después: } \\
\text { ral en el GI. } \\
\text { yismos resultados que en } \\
\text { - Sin efectos significativos entre el el post-test. } \\
\text { GI y GC en el aspecto perceptivo } \\
\text { y conductual de la IC, ya que no } \\
\text { hubieron diferencias en estimar el } \\
\text { tamaño corporal y las conductas } \\
\text { de dieta y vómitos entre ambos } \\
\text { grupos. }\end{array}$ \\
\hline $\begin{array}{l}\text { Girls on the } \\
\text { Go! (GoG) }\end{array}$ & $\begin{array}{l}\text { Tirlea et al. } \\
(2016)\end{array}$ & $\begin{array}{l}N=122+\text { de No } \\
10-16 \text { años. } \\
n=60 \text { q de } 10- \\
12 \text { años. }\end{array}$ & $\begin{array}{l}\text { IC, autoestima, se- } \\
\text { guridad personal y } \\
\text { autoafirmación, men- } \\
\text { te saludable, AF, AS, } \\
\text { confianza y RI. } \\
\text { Categorías: } 2,3,5,6 \\
\text { y } 7 \text {. }\end{array}$ & $\begin{array}{l}10 \text { sesiones. } 1^{2} \\
\text { y } 10^{a} \text { sesión: } \\
\text { 1h. } \\
\text { Resto: } 3 \mathrm{~h} . \\
\text { Total: } 26 \mathrm{~h} .\end{array}$ & a - Mejoras en autoestima. \\
\hline $\begin{array}{l}\text { Beautiful } \\
\text { from the } \\
\text { inside out }\end{array}$ & $\begin{array}{l}\text { Norwood, } \\
\text { Murray, } \\
\text { Nolan y } \\
\text { Bowker } \\
(2011)\end{array}$ & $\begin{array}{l}N=77 \text { niños No } \\
\text { de } 10-12 \text { ańos. } \\
n=36 \text { ` y } 41 \%\end{array}$ & $\begin{array}{l}\text { Autoestima, AM, } \\
\text { iguales, individuali- } \\
\text { dad, habilidades co- } \\
\text { municativas y belleza } \\
\text { real vs. artificial. } \\
\text { Categorías: } 2,5,6 \text { y } 7 \text {. }\end{array}$ & $\begin{array}{l}5 \text { de } 80 \text { min. } \\
\text { Total: } 6 \text { h. } y \\
40 \text { min. }\end{array}$ & $\begin{array}{l}\text { - Mejora de la satisfacción corporal } \\
\text { en ambos géneros. } \\
\text { y - La estima de la apariencia fue ma- } \\
\text { yor en chicos. } \\
\text { - Disminuyó la presión sentida por } \\
\text { las chicas para obtener el ideal del- } \\
\text { gado, y la de los chicos para el ideal } \\
\text { musculado. }\end{array}$ \\
\hline $\begin{array}{l}\text { Trouble } \\
\text { on the } \\
\text { Tightrope: } \\
\text { In Search of } \\
\text { Skateboard } \\
\text { Sam } \\
\text { EE. UU. }\end{array}$ & $\begin{array}{l}\text { Cousineau } \\
\text { et al. }(2010)\end{array}$ & $\begin{array}{l}N=190 \text { niños Sí } \\
\text { de } 11 \text { y } 12 \text { ańos. } \\
n=82 \text { ○े y } 108 \\
q\end{array}$ & $\begin{array}{l}\text { Pubertad, nutrición, } \\
\text { AF, autoestima, AM } \\
\text { y RI. } \\
\text { Categorías: } 2,3,5 \text { y } 7 \text {. }\end{array}$ & $\begin{array}{l}3 \text { de } 60 \mathrm{~min} . \\
\text { Total: } 3 \mathrm{~h} .\end{array}$ & $\begin{array}{l}\text { - Chicas: mejor autoestima en el GI. } 3 \text { meses después: } \\
\text { - El GI y el GC tuvieron similares - Chicas: mejora de pun- } \\
\text { puntuaciones en el Self-Perception tuaciones totales en el SPP. } \\
\text { Profile (SPP), siendo evaluadas tres -Chicos: mismos resulta- } \\
\text { de sus escalas: aceptación social, dos que en el post-test. } \\
\text { apariencia física y autoestima ge- } \\
\text { neral. } \\
\text { - Chicos: los del GC mejoraron la } \\
\text { autoestima y sus puntuaciones en } \\
\text { el } S P P \text {, mientras que el GI empeo- } \\
\text { ró. }\end{array}$ \\
\hline $\begin{array}{l}\text { Self-esteem } \\
\text { and Healthy } \\
\text { Body Image } \\
\text { Program } \\
\text { Australia }\end{array}$ & $\begin{array}{l}\text { McCabe et } \\
\text { al. (2010) }\end{array}$ & $\begin{array}{l}N=421 \delta^{\hat{~ d e ~ S i ́ ~}} \\
11-15 \text { años. }\end{array}$ & $\begin{array}{l}\text { Cualidades personales, } \\
\text { autoestima, RI, habi- } \\
\text { lidades comunicativas, } \\
\text { sociales y de afronta- } \\
\text { miento. } \\
\text { Categorías: 2, 4, 5, } 6 \\
\text { y } 7 \text {. }\end{array}$ & $\begin{array}{l}5 \text { de } 60 \mathrm{~min} . \\
\text { Total: } 5 \mathrm{~h} .\end{array}$ & $\begin{array}{l}\text { - No hubieron diferencias entre el 3,6 y } 12 \text { meses después: } \\
\text { GI y GC en InC, estrategias de - Reducción significativa } \\
\text { cambio corporal, afecto negativo, del afecto negativo. } \\
\text { autoestima y RI. }\end{array}$ \\
\hline
\end{tabular}




\begin{tabular}{|c|c|c|c|c|c|}
\hline \multirow{2}{*}{$\begin{array}{c}\text { Programa y } \\
\text { país/es }\end{array}$} & \multirow{2}{*}{$\begin{array}{c}\text { Autor/es y } \\
\text { año }\end{array}$} & \multirow{2}{*}{$\begin{array}{l}\text { Muestra total y GC } \\
\text { subgrupos }\end{array}$} & \multirow{2}{*}{$\begin{array}{l}\text { Contenidos y cate- } \\
\text { gorías }\end{array}$} & \multirow{2}{*}{$\begin{array}{l}\text { Sesiones y } \\
\text { duración }\end{array}$} & Resultados \\
\hline & & & & & Post-intervención \\
\hline $\begin{array}{l}\text { MS Body } \\
\text { Project } \\
\text { EE.UU. }\end{array}$ & $\begin{array}{l}\text { Rohde et al. } \\
(2014)\end{array}$ & $\begin{array}{l}N=81 \text { q de Sí } \\
11-14 \text { años (es- } \\
\text { tudio } 1 \text { ). } \\
N=52 q \text { de } 11- \\
14 \text { años (estu- } \\
\text { dio 2). }\end{array}$ & $\begin{array}{l}\text { IA, autoafirmación, } \\
\text { dieta, activismo cor- } \\
\text { poral, IC y ConA. } \\
\text { Categorías: } 2,3 \text { y } 6 \text {. }\end{array}$ & $\begin{array}{l}6 \text { de } 45 \text { min. } \\
\text { Total: } 4 \text { h. y } \\
30 \text { min. }\end{array}$ & $\begin{array}{l}\text { - GI (estudio 1): reducción signifi- } 3 \text { meses después (en parti- } \\
\text { cativa de la presión percibida para cipantes del estudio 2): } \\
\text { ser delgadas; pequeño-mediano Ningún efecto significa- } \\
\text { efecto en IID, InC, dieta y sínto- tivo. } \\
\text { mas de desórdenes alimentarios; } \\
\text { y ninguna repercusión en afecto } \\
\text { negativo. } \\
\text { - GI (estudio 2): efecto medio en el } \\
\text { afecto negativo y sin efecto signifi- } \\
\text { cativo en el resto de variables. }\end{array}$ \\
\hline $\begin{array}{l}\text { Body } \\
\text { Image in } \\
\text { the Primary } \\
\text { School }\end{array}$ & $\begin{array}{l}\text { Hutchinson } \\
\text { y Calland } \\
(2011) \text {. }\end{array}$ & $\begin{array}{l}N=140 \text { niños Sí } \\
\text { de } 9 \text { y } 10 \text { ańos. } \\
n=70 \lesssim \text { y } 70 \text { ? }\end{array}$ & $\begin{array}{l}\text { Diversidady valora- } \\
\text { ción de apariencias, } \\
\text { gestión de burlas y } \\
\text { desarrollo de resilien- } \\
\text { cia a las presiones de } \\
\text { medios e iguales sobre } \\
\text { apariencia. } \\
\text { Categorías: } 1,2 \text { y } 6 \text {. }\end{array}$ & 6 de $60 \mathrm{~min}$. & $\begin{array}{l}\text { - Chicas del GI: mejor autoestima. } 3 \text { meses después: } \\
\text { Sin diferencias entre grupos en IIB,- Chicas del GI: mejor au- } \\
\text { conciencia de los IA y presión me- toestima. } \\
\text { diática percibida. } \\
\text { - Chicos: ligera mejora en IIB. Sin la IIB. } \\
\text { diferencias entre GI y GC en au-- Ambos géneros: mayor } \\
\text { toestima, conciencia de los IA y conciencia de la manipu- } \\
\text { presión mediática percibida. lación mediática de imá- } \\
\text { - Ambos géneros: mayor conciencia genes. } \\
\text { de la manipulación mediática de } \\
\text { imágenes. }\end{array}$ \\
\hline
\end{tabular}

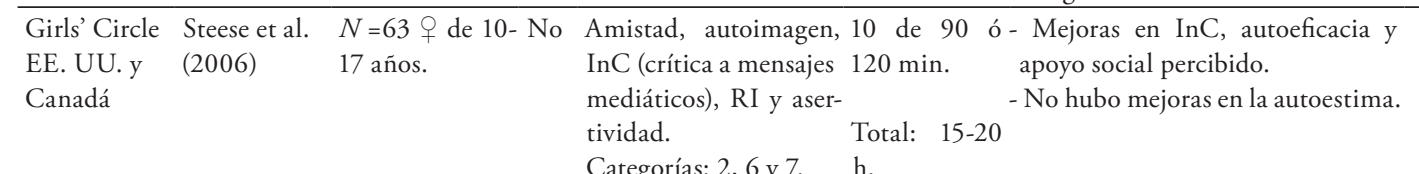

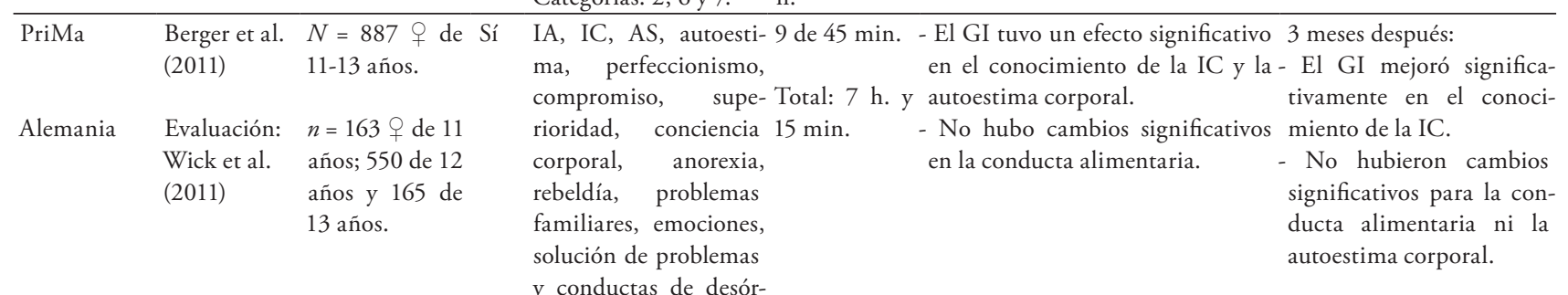
y conductas de desórdenes alimentarios. Categorías: 1, 2, 3, 4, 5 y 6.

Very Impor- Haines, $\quad N=151$ niños Sí - Nivel individual: IC, 10 sesiones, 8 meses después:

tant Kids Neumark- de 9-11 ańos, habilidades para lidiar precedidas - La prevalencia de los insultos por Sztainer, Pe- padres y profe- insultos sobre peso, por $1 \mathrm{~h}$. de AF apariencia o peso aumentó de un rry, Hannan sores. $\quad$ AM, AS y AF. (programa 21.1\% a un 29.8\% en el GC. Por

EE.UU. y Levine - Nivel contextual: IC extra esco-el contrario, en el GI disminuyó

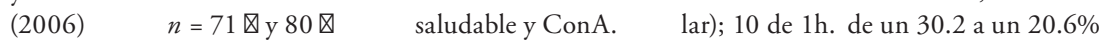

- Nivel familiar: insul- (programa de - El GI mejoró la autoeficacia hacia tos, impacto mediático teatro) y 2 se- el impacto de las normas de insulen IC y ser físicamente siones de pro- tos sobre el peso y disminuyó las activo con su familia. grama con la normas negativas de iguales relaCategorías: 2,3 y 6 . familia. cionadas con el peso. Total: $32 \mathrm{~h}$.

Healthy Stock et al. $\quad N=383$ ô de Sí AS, AF e IC saludable. 2-3 h/sem.,- De 5 a 8 años: el GI tuvo menor Buddies (2007) 5-12 años. Categorías: 1,3 y 6. $21 \mathrm{sem} .(8-12$ aumento de presión sanguínea y

$\begin{array}{ll}\text { Canadá } & \text { años). Éstos mejoras en actitudes saludables. } \\ \text { enseñaban En el GI y GC aumentaron los }\end{array}$ enseñaban En el GI y GC aumentaron los durante 30 comportamientos saludables y los min. a los pe- conocimientos sobre salud. queños. To-- De 8 a 12 años: el GI tuvo mados: 60 min. yor conocimiento sobre salud, más de AF/sem. comportamientos y actitudes saludables. 


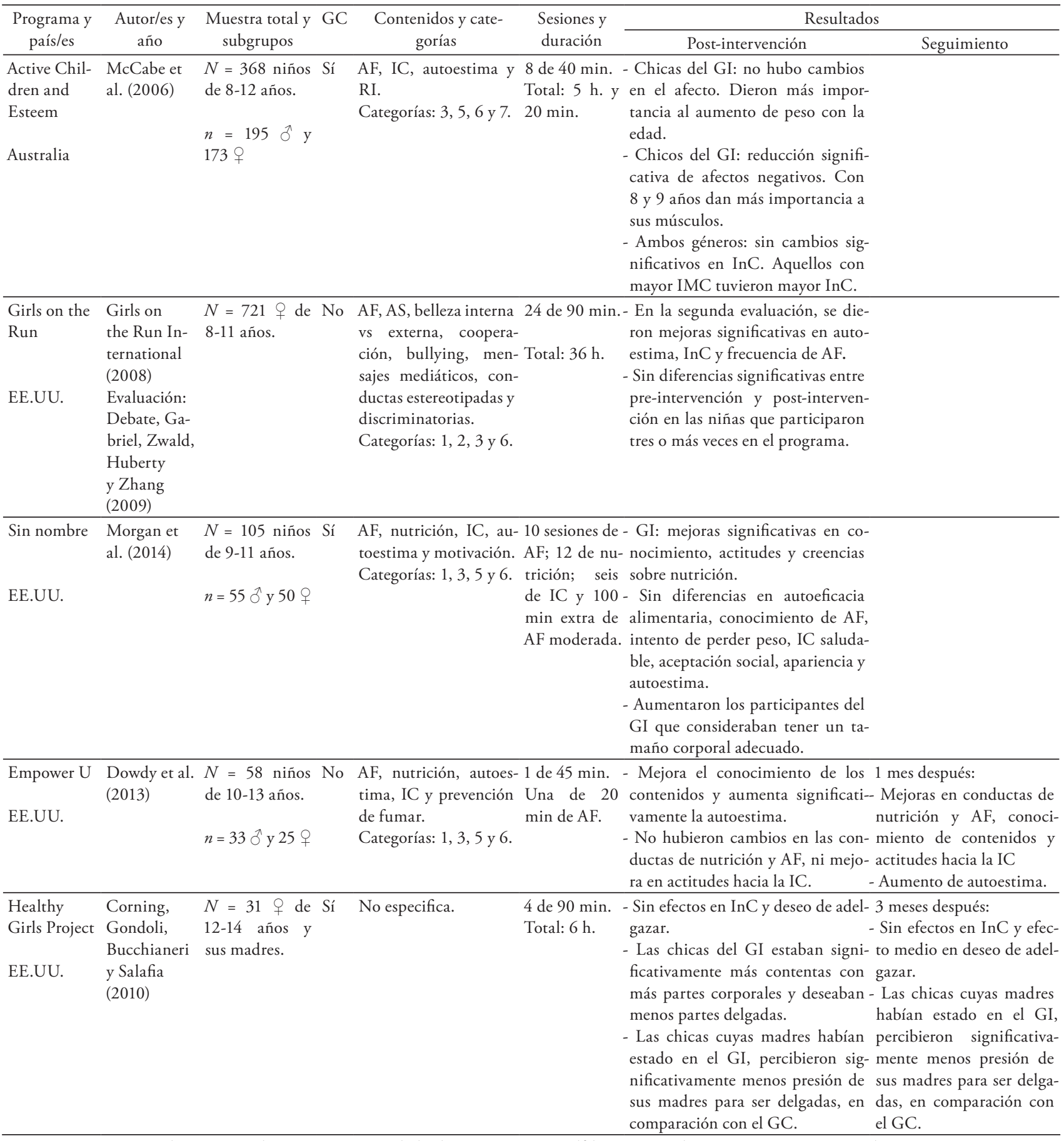

Nota GC = Grupo Control, GI = Grupo de Intervención, IA = Ideales de Apariencia, AM = Alfabetización Mediática, CA = Comparaciones sobre Apariencia, ConA = Conversaciones sobre Apariencia, RI = Relaciones con Iguales, InC = Insatisfacción corporal, IIB = Internalización de Ideales de Belleza, IID = Internalización del Ideal Delgado, RA = Restricción Alimentaria, AS = Alimentación Saludable. 
Tabla 2. Resumen de resultados en el post-test y el seguimiento de las variables más representativas

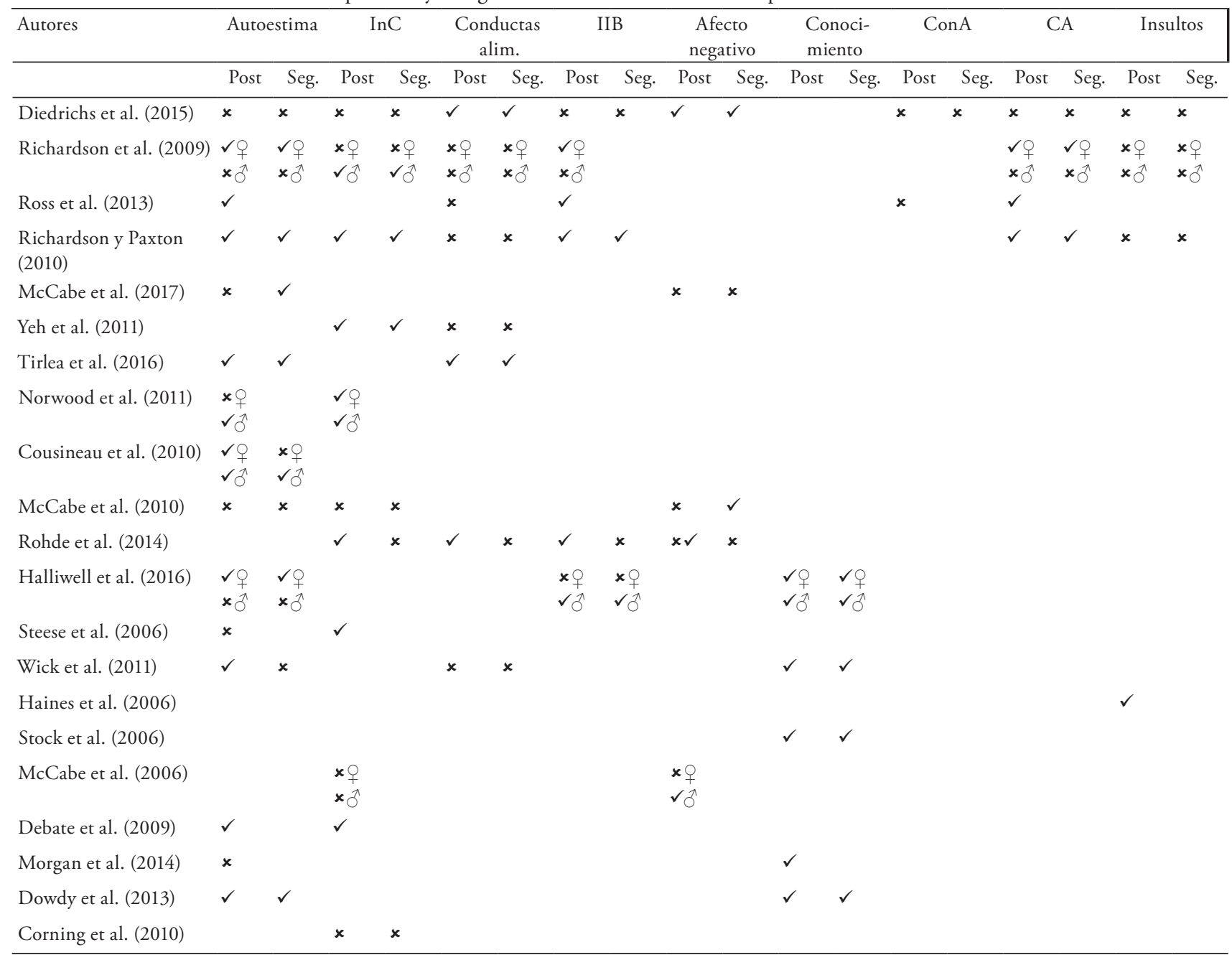

Nota: No se especifica símbolo de género cuando los participantes son de un solo género o los resultados son iguales para chicos y chicas. No existen mejoras significativas ( $\mathbf{x}$; existen mejoras significativas $(\checkmark)$

\section{Características generales de los estudios}

De los 21 programas incluidos, 11 están dirigidos a ambos géneros, ocho de ellos sólo a chicas y los dos restantes, al género masculino.

En cuanto a los países, aquellos en los que más prevalece el desarrollo de programas son EE.UU. con ocho programas, y Australia, con seis. No obstante, también se ha incluido uno en China, Inglaterra, Reino Unido y Alemania, dos en Canadá y uno en EE.UU. y Canadá conjuntamente.

\section{Otras características de los programas}

Entre los objetivos de los programas destacan el aumento de autoestima y alfabetización mediática, la promoción y aumento de una alimentación y AF saludables, la reducción de la internalización de ideales de belleza, la prevención de actitudes y conductas relacionadas con los TCA, y la disminución de conversaciones o insultos sobre apariencia.

En relación a la metodología, muchos programas optan por un diseño interactivo (Corning et al., 2010; Cousineau et al., 2010; Diedrichs et al., 2015; Dowdy et al., 2013; Haines et al., 2006; Halliwell et al., 2016; McCabe et al., 2010; McCabe et al., 2017; Norwood et al., 2011; Richardson et al., 2009; Richardson \& Paxton, 2010; Steese et al., 2006; Tirlea et al., 2016: Yeh et al., 2011) donde los niños participan en grupos de discusión, role play, juegos, vídeos y otras actividades grupales. El resto de programas eligen una metodología mixta, incorporando también sesiones didácticas.

Respecto a los enfoques, algunos autores defienden y llevan a cabo un enfoque etiológico (Diedrichs et al., 2015; Richardson \& Paxton, 2010) o socio-cognitivo (McCabe et 
al., 2017); otros, un enfoque ecológico (Corning et al., 2010; Haines et al., 2006) y otros, no especifican el enfoque en el que se basa el programa diseñado.

La implementación de estos programas suelen hacerla los propios investigadores, aunque en algunos casos los encargados son los profesores (Diedrichs et al., 2015; Morgan et al., 2014), algunos estudiantes graduados en enfermería (Dowdy et al., 2013) y en psicología (Norwood et al., 2011) o profesionales de la salud (Tirlea et al., 2016).

Finalmente, respecto a la duración de los programas, se ha observado que la mayoría suelen oscilar entre tres y seis horas, siendo de 90 minutos el más breve (Diedrichs et al., 2015). Además, es el único programa que cuenta con una sola sesión, mientras que los demás son de multisesiones (una o dos por semana).

\section{Efectos en la post-intervención}

Los resultados suelen reflejar ligeros o inexistentes efectos en los índices de insatisfacción corporal, en las conductas o síntomas de desórdenes alimentarios y en los insultos sobre apariencia. Once programas evalúan la repercusión en la insatisfacción, aunque tres no hallan mejora (Corning et al., 2010; Diedrichs et al., 2015; McCabe et al., 2006; McCabe et al., 2010), dos tienen un efecto pequeńo-moderado (Richardson et al., 2009; Rohde et al., 2014), aunque éste último sólo en los chicos, y los seis restantes obtienen mejoras significativas (Tabla 2). En cuanto a las conductas o síntomas de desórdenes alimentarios, sólo tres de ocho programas demuestran un efecto significativo (Diedrichs et al., 2015; Rohde et al., 2014; Yeh et al., 2011). Por otro lado, se hallan efectos insignificantes en los insultos sobre apariencia, ya que de los cuatro estudios que lo analizan en el post-test y seguimiento, sólo uno disminuye los insultos (Haines et al., 2006).

En relación a la AF, los dos programas que trabajan este contenido y evalúan el nivel de descontento corporal, muestran mejoras en la insatisfacción (Debate et al., 2009; McCabe et al., 2006). Por otro lado, de los seis programas de AF que evalúan la autoestima, cuatro de ellos aumentan los niveles de ésta (Cousineau et al., 2010; Debate et al., 2009; Tirlea et al., 2016).

Respecto a la internalización de los ideales de belleza, cuatro de los cinco programas que evalúan esta variable, mejoran también la insatisfacción (Dunstan et al., 2016; Norwood et al., 2011; Richardson \& Paxton, 2010; Rohde et al., 2014), aunque sólo uno lo hace en los síntomas bulímicos o de desórdenes alimentarios (Rohde et al., 2014).

De los 21 programas incluidos en la revisión, 15 evalúan la autoestima y de éstos, 10 presentan mejoras en el post-test, aunque sólo tres lo hacen en uno de los géneros. Esta mejoría en autoestima no es mantenida a lo largo del tiempo en todos los casos.

\section{Efectos en el seguimiento}

Once de los 13 programas que incluyen una evaluación de seguimiento la hacen tres meses después de la intervención; uno de ellos, un mes después; y otro, entre cuatro y 9.5 semanas tras acabar el programa. De los 10 que realizan la evaluación tres meses después, uno vuelve a replicar dicha evaluación a los seis meses (Tirlea et al., 2016); y otro estudio, a los seis y 12 meses (McCabe et al., 2010).

Los efectos de los programas a largo plazo son menos notables. Un total de ocho estudios evalúan la insatisfacción un tiempo después de haber finalizado la intervención, pero sólo tres tienen mejoras en esta variable (Richardson et al., 2009; Richardson y Paxton, 2010; Yeh et al., 2011). De los programas que mejoraban la satisfacción de sus participantes tras la intervención, sólo hay uno que no mantiene esta mejoría un tiempo después (Rohde et al., 2014).

Respecto a las conductas o síntomas de desórdenes alimentarios, únicamente un programa conserva las mejoras,de cuatro a nueve semanas después de la intervención (Diedrichs et al., 2015); y otro, tiene efectos en el seguimiento, pero no tras la intervención (Tirlea et al., 2016).

De los 10 programas que apreciaron mejora en la autoestima en el post-test, dos no la mantienen un tiempo después (Diedrichs et al., 2015; Wick et al., 2011) y dos no evalúan este seguimiento. Por el contrario, el programa desarrollado por McCabe et al. (2017) sólo tiene efectos positivos en la autoestima tres meses después, pero no en el post-test.

En líneas generales, sólo dos programas tuvieron los mismos efectos en todas las variables, tanto en el post-test como en el seguimiento (Richardson \& Paxton, 2010; Yeh et al., 2011).

\section{Discusión}

El propósito de este estudio fue analizar los programas diseñados para la mejora de la IC en niños de Educación Primaria, a fin de conocer los contenidos que más suelen emplearse en dichos programas, los que pueden tener mayor efecto en los participantes y las limitaciones de éstos.

En relación a las variables evaluadas, en las Tablas 1 y 2 puede observarse que las mejoras en insatisfacción corporal y síntomas de desórdenes alimentarios no son demasiado evidentes, tal y como apreciaron Holt y Ricciardelli (2008) en su revisión de programas. Por tanto, las intervenciones existentes podrían no ser totalmente efectivas para tal fin.

Además de estas variables, se evaluaron otras como alfabetización mediática e internalización de los ideales de belleza, las cuales son relevantes en el currículum de Primaria, dentro del área de Educación Física (Real Decreto 126/2014). Los efectos en dicha internalización, tanto del ideal delgado como musculado, son evidentes en muchas ocasiones, aun- 
que el ideal musculado sólo es evaluado en un programa (Richardson et al., 2009). En relación a esta internalización, Stice, Chase, Stormer, \& Appeal (2001) sostienen que, si un programa de prevención reduce la internalización del ideal delgado, probablemente conseguirá una disminución en otros factores de riesgo como la insatisfacción corporal, dieta, afecto negativo y síntomas bulímicos, como así lo demuestran algunos programas analizados (Richardson \& Paxton, 2010; Rohde et al., 2014; Ross et al., 2013).

Para la evitación o mejora de esta internalización es necesario abordar la alfabetización mediática desde edades tempranas, puesto que los nińos son continuos receptores de la información que transmiten los medios. Además, también es importante reducir el tiempo de exposición a éstos, ya que este hecho predice que los niños tengan una IC negativa y mayores desórdenes alimentarios (Levine \& Chapman, 2012). Sobre ello, Tiggemann y Slater (2014) comprobaron que el tiempo que las niñas preadolescentes pasaban en internet estaba asociado con un aumento de la preocupación con su IC, la internalización del ideal delgado y la dieta, así como una disminución de la estima corporal.

Por otro lado, existen programas que hacen hincapié en las relaciones sociales con los iguales, ya que a través de ellas se puede frenar la presión social sobre la IC (Littleton \& Oilendick, 2003). Por consiguiente, el desarrollo de habilidades sociales se hace fundamental entre los niños (Saiz, Prieto, Gutiérrez, \& Gil, 2016). Unas adecuadas relaciones interpersonales suponen una buena herramienta para frenar los insultos y discriminaciones sobre la apariencia, ya que ésta es la forma más común de ofenderse entre los niños, especialmente el género masculino (Smolak, 2004). A este respecto, el peso es considerado por los estudiantes como el principal motivo de exclusión y victimización (Puhl, Luedicke, \& Heuer, 2011).

Los prejuicios y conversaciones sobre apariencia se deben a que los niños suelen compararse con otros y autoevaluarse según su apariencia, habilidades físicas, competencia académica y social (Smolak, 2012). Por ello, las conversaciones y comparaciones sobre apariencia necesitan ser consideradas en las intervenciones, para evitar que la IC y el autoconcepto se vean dańados por dichas comparaciones.

Los programas analizados en este estudio no reflejan disminución de dichas conversaciones, a excepción de un estudio (Richardson \& Paxton, 2010). En cuanto a las comparaciones de apariencia, todos los programas menos uno (Diedrichs et al., 2015) mostraron una disminución en esta variable, aunque Richardson et al. (2009) comprobaron que esta mejora sólo se daba en niñas. No obstante, ésta no es igual de observable en relación a los insultos sobre apariencia, puesto que de los estudios que analizan esta variable, sólo el 25\% halla una disminución (Haines et al., 2006). Otro de los contenidos tratado es la alimentación saludable (Ge- hman et al., 2006; Haines et al., 2006; McCabe et al., 2017; Tirlea et al., 2016), aunque Hutchinson y Calland (2011) consideran que hablarles a los niños de alimentación al abordar la IC puede ser contraproducente, ya que podría ocasionarles ansiedades o preocupaciones con la alimentación y el peso, que quizá desencadenarían en desórdenes alimentarios.

Por otro lado, y vinculada con la alimentación, aparece la AF. Ésta es considerada en los programas como medio para alcanzar una IC saludable, ya que es sabido que la práctica de ésta se convierte en un factor protector frente al desarrollo de una IC negativa (Littleton \& Oilendick, 2003; López, 2017; López, Díaz, \& Smith, 2018; López, López, \& Díaz, 2015). De hecho, en la revisión realizada, la AF tiene una notable repercusión en la mejora de la insatisfacción corporal y la autoestima; aunque la apreciación del cuerpo sólo se asocia positivamente con la AF cuando la motivación del ejercicio no radica en la apariencia (Homan \& Tylka, 2014), puesto que existen deportes como danza, patinaje o gimnasia, que focalizan en el aspecto físico y la delgadez por razones estéticas (Krahnstoever, Earnest, \& Birch, 2002).

Teniendo en consideración la incidencia del ejercicio en la IC, Martin y Bassett (2012) realizan unas recomendaciones para desarrollar programas de AF que mejoren la IC. Consideran que el ejercicio debe ser frecuente y de intensidad moderada-vigorosa; las actividades tienen que ser lúdicas, ya que ello se asocia con cambios en la IC; hay que incidir en los beneficios para la salud y no considerar el ejercicio como vía para cambiar la apariencia; se deben proponer metas alcanzables y enseñarles a controlar su mejora de la condición física, es decir, su progreso en términos funcionales y no de apariencia.

\section{Limitaciones y prospectivas}

Los estudios analizados cuentan con algunas limitaciones. Una de ellas es la falta de un grupo control (Debate et al., 2009; Dowdy et al., 2013; Norwood et al., 2011; Steese et al., 2006; Tirlea et al., 2016) que permita comparar los efectos de la intervención entre éste y el experimental. Por consiguiente, no puede observarse la eficacia del programa en su totalidad (Holt \& Ricciardelli, 2008).

Asimismo, ocho programas no realizan una evaluación de seguimiento para observar si los efectos del programa se mantienen en el tiempo y si éste es efectivo. La mayoría de estudios que realizan este seguimiento, suelen hacerlo tres meses después.

Otra limitación ha sido la muestra pequeña (Norwood et al., 2011; Rohde et al., 2014; Ross et al., 2013; Steese et al., 2006), por lo que es necesario aplicar algunos programas con más participantes.

En vistas a los futuros programas, podría ser adecuado incluir la AF como contenido, ya que según lo observado en 
el trabajo, ésta parece tener un impacto positivo en la insatisfacción corporal y la autoestima. Por tanto, convendría complementar las actividades del aula con diversas sesiones de AF, con el fin de reforzar y hacer más eficaz el trabajo de la IC. Asimismo, también sería importante diseñar y llevar a cabo programas con niños de edades más tempranas, antes de que formen una IC negativa.

\section{Conclusiones}

Este estudio refleja una visión general de los programas diseńados más recientemente para la mejora de la IC, lo cual ofrece un amplio recurso de estrategias y contenidos que pueden desarrollarse en las aulas para mejorar la IC de los niños. Los resultados reflejan que los cambios más significativos se dan en el conocimiento sobre el tema de la IC y la autoestima. Sin embargo, esto no ocurre en la insatisfacción corporal, los insultos sobre apariencia y los síntomas de desórdenes alimentarios, donde el efecto de los programas es generalmente nulo o pequeño-moderado. Por otro lado, es destacable la influencia de la AF en la insatisfacción corporal y la autoestima de los niños, ya que los estudios que incluyeron AF y evaluaron la insatisfacción mostraron mejoras en esta variable y en la autoestima. Asimismo, la mejora en la internalización de los ideales de belleza también parecía repercutir positivamente en la insatisfacción, aunque no los síntomas bulímicos o de desórdenes alimentarios.

Otro aspecto relevante observado en este trabajo es que algunas mejoras que se dan tras la intervención suelen perderse en la evaluación de seguimiento, por lo que algunos programas parecen no ser efectivos a largo plazo. En este sentido, podría ser interesante desarrollar nuevos programas de mayor duración o que incluyan a padres (McCabe et al., 2017), ya que abordando la intervención desde un enfoque ecológico que incluya a diferentes agentes del entorno del nińo, los efectos positivos de los programas podrían perdurar con el tiempo.

\section{Referencias}

1. American Psychiatric Association (2014). Guía de consulta de los criterios diagnósticos del DSM-5. Arlington: Author

2. Bird, E. L., Halliwell, E., Diedrichs, P. C., \& Harcourt, D. (2013). Happy Being $\mathrm{Me}$ in the UK: A controlled evaluation of a school-based body image intervention with pre-adolescent children. Body image, 10(3), 326-334. doi: 10.1016/j.bodyim.2013.02.0086

3. Cash, T. F. (2012). Cognitive-behavioral perspectives on body image. En T. Cash \& L. Smolak (Eds.), Body image. A handbook of science, practice and prevention (pp. 39-47). New York: Guilford Publications.

4. Corning, A. F., Gondoli, D. M., Bucchianeri, M. M., \& Salafia, E. H. B. (2010). Preventing the development of body issues in adolescent girls through intervention with their mothers. Body Image, 7(4), 289-295. doi: 10.1016/j.bodyim.2010.08.001

5. Couisenau, T. M., Franko, D. L., Trant, M., Rancourt, D., Ainscough, J., Chaudhuri, A., \& Brevard, J. (2010). Teaching adolescents about changing bodies: Randomized controlled trial of an Internet puberty education and body dissatisfaction prevention program. Body image, 7(4), 296-300. doi: 10.1016/j.bodyim.2010.06.003

6. Damiano, S., Gregg, K., Spiel, E., McLean, S., Wertheim, E., \& Paxton, S. (2015). Relationships between body size attitudes and body image of 4-year-old boys and girls, and attitudes of their fathers and mothers. Journal of Eating Disorders,3(16), 1-10. doi: 10.1186/s40337-015-00480

7. Debate, R. D., Gabriel, K. P., Zwald, M., Huberty, J., \& Zhang, Y. (2009). Changes in psychosocial factors and physical activity frequency among third- to eighth-grade girls who participated in a developmentally focused youth sport program: a preliminary study. Journal of School Health, 79(10), 474-484. doi: 10.1111/j.1746-1561.2009.00437.x

8. Diedrichs, P. C., Atkinson, M. J., Steer, R. J., Garbett, K. M., Rumsey, N., \& Halliwell, E. (2015). Effectiveness of a brief school-based body image intervention 'Dove Confident Me: Single Session' when delivered by teachers and researchers: Results from a cluster randomized controlled trial. Behaviour Research and Therapy, 74, 94-104. doi: 10.1016/j. brat.2015.09.004

9. Dowdy, S., Alvarado, M., Atieno, O., Barker, S., Barrett, S., Carlton A.,...\&Williamson, L. (2013). Empower U: Effectiveness of an adoles- cent outreach and prevention program with sixth-grade boys and girls: A pilot study. Journal of Pediatric Nursing, 28, 77-84. doi: 10.1016/j. pedn.2012.03.028

10. Evans, R. R., Roy, J., Geiger, B. F., Werner, K. A., \& Burnett, D. (2008). Ecological strategies to promote healthy body image among children. Journal of School Health, 78(7), 359-367. doi: 10.1111/j.17461561.2008.00315.x

11. Ferreiro, F., Seoane, G., \& Senra, C. (2014). Toward understanding the role of body dissatisfaction in the gender differences in depressive symptoms and disordered eating: A longitudinal study during adolescence. Journal of Adolescence, 37(1), 73-84. doi: 10.1016/j.adolescence.2013.10.013

12. González-Martí, I., Fernández, J. G., Hernández-Martínez, A., \& Contreras, O. R. (2014). Physical perceptions and self-concept in athletes with muscle dysmorphia symptoms. The Spanish Journal of Psychology, 17(e43), 1-7. doi: 10.1017/sjp.2014.45

13. Grogan, S. (2016). Body image. Understanding body dissatisfaction in men, women and children. London: Routledge.

14. Haines, J., Neumark-Sztainer, D., Perry, C. L., Hannan, P. J., \& Levine, M. P. (2006). V.I.K. (Very Important Kids): a school-based program designed to reduce teasing and unhealthy weight-control behaviors. Health Education Research, 21(6), 884-895. doi: 10.1093/her/cyl123

15. Halliwell, E., Yager, Z., Paraskeva, N., Diedrichs, P. C., Smith, H., \& White, P. (2016). Body Image in Primary Schools: A pilot evaluation of a primary school intervention program designed by teachers to improve children's body satisfaction. Body Image, 19, 133-141. doi: 10.1016/j. bodyim.2016.09.002

16. Harriger, J. A., Calogero, R. M., Witherington, D., \& Smith, J. E. (2010). Body size stereotyping and internalization of the thin ideal in preschool girls. Sex Roles, 63, 609-620. doi: 10.1007/s11199-010-9868-

17. Holt, K. E., \& Ricciardelli, L. A. (2008). Weight concerns among elementary school children: A review of prevention programs. Body image, 5(3), 233-243. doi: 10.1016/j.bodyim.2008.02.002

18. Homan, K. J., \& Tylka, T. L. (2014). Appearance-based exercise motivation moderates the relationship between exercise frequency and 
positive body image. Body Image, 11(2), 101-108. doi: 10.1016/j.bodyim.2014.01.003

19. Hutchinson, N., \& Calland, C. (2011). Body image in the primary school. Londres: Taylor \& Francis Group.

20. Krahnstoever, K. K., Earnest, M. B., \& Birch, L. L. (2002). Participation in aesthetic sports and girls' weight concerns at ages 5 and 7 years. International Journal of Eating Disorders, 31(3), 312-317. doi: 10.1002/ eat. 10043

21. Levine, M. P., \& Chapman, K. (2012). Media influencers on body image. En T. Cash \& L. Smolak (Eds.), Body image. A handbook of science, practice and prevention (pp. 101-109). New York: Guilford Publications

22. Levine, M. P., \& Smolak, L. (2006). The prevention of eating problems and eating disorders. Theory, research, and practice. Mahwan, Nueva Jersey: Psychology Press, Lawrence Erlbaum Associates.

23. Littleton, H. L., \& Oilendick, T. (2003). Negative body image and disordered eating behavior in children and adolescents: what places youth at risk and how can these problems be prevented? Clinical Child and Family Psychology Review, 6(1), 51-66. doi: 10.1023/A:1022266017046

24. López-Sánchez, G. F. (2017). Composición corporal, imagen corporal, actividad física y salud en niños y adolescentes (Tesis Doctoral, Universidad de Murcia). Recuperada de http://www.tesisenred.net/handle/10803/401819

25. López Sánchez, G. F., Díaz Suárez, A., \& Smith, L. (2018). Analysis of body image and obesity by Stunkard's silhouettes in 3- to 18-year-old Spanish children and adolescents. Anales de psicología, 34(1), 167-172. doi: 10.6018/analesps.34.1.294781

26. López-Sánchez, L., López-Sánchez, G. F., \& Díaz-Suárez, A. (2015). Efectos de un programa de actividad física en la imagen corporal en escolares con TDAH. Cuadernos de Psicología del Deporte, 15(2), 135-142.

27. Martin, K. A., \& Bassett, R. L. (2012). Exercise and changes in body image. En T. F. Cash y L. Smolak (Eds.), Body image. A handbook of science, practice and prevention (pp. 378-393). New York: Guilford Publications.

28. McCabe, M. P., Ricciardelli, L. A, \& Salmon, J. O. (2006). Evaluation of a prevention program to address body focus and negative affect among children. Journal of Health Psychology, 11(4), 589-598. doi: $10.1177 / 1359105306065019$

29. McCabe, M., P., Connaughton, C., Tatangelo, G., \& Mellor, D. (2017). Healthy me: A gender-specific program to address body image concerns and risk factors among preadolescents. Body image, 20, 20-30. doi: 10.1016/j.bodyim.2016.10.007

30. McCabe, M., P., Ricciardelli, L. A., \& Karantzas, G. (2010). Impact of a healthy body image program among adolescent boys on body image, negative affect, and body change strategies. Body image, 7(2), 117-123. doi: 10.1016/j.bodyim.2009.10.007

31. Morgan, A. Z., Ulrich, P., Simmons, K. P., Gropper, S. S., Jo Connell, L. J., Daniels, M. K.,... \& Keiley, M. K. (2014). Effectiveness of a multi-faceted, school-based health intervention program with 4th graders in Alabama. Children and Youth Services Review, 37,46-54. doi: 10.1016/j.childyouth.2013.12.006

32. Norwood, S. J., Murray, M., Nolan, A., \& Bowker, A. (2011). Beautiful from the inside out: A school-based programme designed to increase self-esteem and positive body image among preadolescents. Canadian Journal of School Psychology, 26(4), 263-282. doi: $10.1177 / 0829573511423632$

33. Puhl, R. M., Luedicke, J., \& Heuer, C. (2011). Weight-based victimization toward overweight adolescents: observations and reactions of peers. Journal of School Health, 81(11), 696-703. doi: 10.1111/j.17461561.2011.00646.x.

34. Raich, R. M. (2001). Imagen corporal. Conocer y valorar el cuerpo. Madrid: Pirámide.
35. Raich, R. M. (2004). Una perspectiva desde la psicología de la salud de la imagen corporal. Avances en Psicología Latinoamericana, 22(1), 15-27.

36. Real Decreto 126 de 2014. Por el que se establece el currículo básico de la Educación Primaria.Febrero 28 de 2014.

37. Richardson, S. M., \& Paxton, S. J. (2010). An evaluation of a body image intervention based on risk factors for body dissatisfaction: A controlled study with adolescent girls. International Journal of Eating Disorders, 43(2), 112-122. doi: 10.1002/eat.20682

38. Richardson, S. M., Paxton, S. J., \& Thompson, J. S. (2009). Is Body Think an efficacious body image and self-esteem program? A controlled evaluation with adolescents. Body Image, 6(2), 75-82. doi: 10.1016/j. bodyim.2008.11.001

39. Rohde, P., Auslander, B. A., Shaw, H., Raineri, K. M., Gau, J. M., \& Stice, E. (2014). Dissonance-based prevention of eating disorder risk factors in middle school girls: results from two pilot trials. International Journal of Eating Disorders, 47(5), 483-494. doi: 10.1002/eat.22253

40. Ross, A., Paxton, S. J., \& Rodgers, R. F. (2013). Y's Girls: Increasing body satisfaction among primary school girls. Body Image, 10(4), 614618. doi: 10.1016/j.bodyim.2013.06.009

41. Saiz, M., Prieto, A., Gutiérrez, E. C., \& Gil, P. (2016). Evaluación inicial en $6^{\circ}$ curso de primaria de las habilidades sociales y propuesta didáctica de intervención en la Educación Física escolar. SPORT TKRevista EuroAmericana de Ciencias del Deporte, 5(1), 65-74.

42. Smolak, L. (2012). Body image development in childhood. En T. Cash \& L. Smolak (Eds.), Body Image. A handbook of science, practice and prevention (pp. 67-75). New York: Guilford Publications.

43. Steese, S., Dollette, M., Phillips, W., Hossfeld, E., Matthews, G., \& Taormina, G. (2006). Understanding girls' circle as an intervention of perceived social support, body image, self-efficacy, locus of control and self-esteem. Adolescence, 41(161), 55-74.

44. Stice, E., Chase, A., Stormer, S., \& Appel, A. (2001). A randomized trial of a dissonance-based eating disorder prevention program. Eating Disorders, 29(3), 247-262. doi: 10.1002/eat.1016

45. Stice, E., \& Shaw, H. (2004). Eating disorder prevention programs: A meta-analytic review. Psychological Bulletin, 130(2), 206-227. doi: 10.1037/0033-2909.130.2.206

46. Stock, S., Miranda, C., Evans, S., Plessis, S., Ridley, J., Yeh, S., \& Chanoine, J. (2007). Healthy Buddies: A novel, peer-led health promotion program for the prevention of obesity and eating disorders in children in elementary school. Pediatrics, 120(4), 1059-1068. doi: 10.1542/ peds.2006-3003

47. Tiggemann, M. (2012). Sociocultural perspectives on human appearance and body image. En T. Cash \& L. Smolak (Eds.), Body image. $A$ handbook of science, practice and prevention (pp. 12-19). New York: Guilford Publications.

48. Tiggemann, M., \& Slater, A. (2014). NetTweens: The internet and body image concerns in preteenage girls. Journal of Early Adolescence, 34(5), 606-620. doi: 10.1177/0272431613501083

49. Tirlea, L., Truby H., \& Haines, T. P. (2016). Pragmatic, randomized controlled trials of the Girls on the Go! Program to improve self-esteem in girls. American Journal of Health Promotion, 30(4), 234-241. doi: $10.1177 / 0890117116639572$

50. Wick, K., Brix, C., Bormann, B., Sowa, M., Strauss, B., \& Berger, U. (2011). Real-word effectiveness of a German school-based intervention for primary prevention of anorexia nervosa in preadolescent girls. Preventive Medicine, 52(2), 152-158. doi: 10.1016/j.ypmed.2010.11.022

51. Yeh, M. C., Liou, Y. M., \& Chien, L. Y. (2011). Development and effectiveness of a school programme on improving body image among elementary school students in Taiwan. Journal of Advanced Nursing, 68(2), 434-443. doi: 10.1111/j.1365-2648.2011.05735.x 